\title{
Ethnobotanical notes on the diversity of tropical wild fruits used by the Adi tribe of Arunachal Pradesh, India
}

\author{
Momang Taram, Hage Yanka, Jambey Tshering ${ }^{1}$ and Hui Tag $^{2}$ \\ Department of Botany, Rajiv Gandhi University, Rono Hills, Doimukh-791112, Arunachal Pradesh, India \\ ${ }^{1}$ Orchid Research Center, Tippi, Balukpong-790114, West Kameng, Arunachal Pradesh, India \\ ${ }^{2}$ Corresponding author, e-mail: huitag2008@gmail.com
}

[Received 18.05.2020; Revised 25.06.2020; Accepted 26.06.2020; Published 30.06.2020]

\begin{abstract}
The objective of the study was to investigate the diversity, local status and bio-cultural importance of tropical wild fruits used among the Adi tribe residing in the East Siang District of Arunachal Pradesh in their traditional biocultural landscape. A total of 88 species representing 56 genera under 39 families have been reported. $87.5 \%$ plants were harvested from wild source and $12.5 \%$ of plants were harvested from semi-domesticated source. The majority of these fruits species were used as food (71 spp.), followed by hunting and fishing ( 9 spp.), Others ( 5 spp.), medicinal (4 spp.) and magico-religious beliefs ( $3 \mathrm{spp}$.). The distribution, IUCN status, and related aspects on sustainable exploitation and strategies for conservation have been discussed.
\end{abstract}

Key words: Arunachal Pradesh, East Siang, Tropical forest, Adi Community, Ethnobotany, Edible Fruits

\section{INTRODUCTION}

Arunachal Pradesh is an Indian state located in the extreme Northeastern corner of the country and is situated on Eastern Himalaya and Indo-Burma hotspots. Topologically it is predominantly hilly and mountainous (Bharali \& Khan 2011) and covers an area of about $83,743 \mathrm{~km}^{2}$, of which $82 \%$ is under forest cover. It is endowed with very rich floral and faunal diversity due to its physiographic variations with elevations ranging from 150 to $6500 \mathrm{~m}$ a.m.s.l. and with unique ambient climatic conditions (Chakravarty et al. 2012; Taram et al. 2018).

The state hosts diverse local culture and dialects spoken by 26 major tribes and 110 subtribes in their traditional Biocultural landscape (Yanka et al. 2019). Of them, the Adi, resident of the Siang belt and Lower Dibang Valley is one of the numerically larger tribes and comprises $26.9 \%$ of the total tribal population of the state (Jha 1985; Krithika et al. 2008). They have mongoloid feature, patriarchal society and with rich heritage of art and craft (Kumar 2015). And are mostly dependent on both domesticated and wild plant resources which are uniquely significant and culturally associated. The use of wild plants is intrinsically linked to their cultural system and is an inseparable component (Angami et al. 2006)

Fruits are the seed-bearing structure of plants which may or may not be edible (Singh \& Asha 2017). Workers like Singh \& Asha (2017) and Lyndoh et al. (2016) had studied the diversity on wild edible fruits of Arunachal Pradesh focused on edibility prospects. Arunachal Pradesh flourishes enormous range of fruits with specific standards of nutritional and medicinal values. However, with the increasing anthropogenic activities due to population explosion and natural calamities over the state, several wild fruits have become vulnerable to their existence (Singh \& Asha 2017). And, therefore, it is feared that if not documented immediately, the ethnobotanically important wild-fruits may face the consequences of being extinct without being recorded. Hence, the present study has tried to unveil the diversity and conservation status of wild fruits used among the Adi community in the tropical vegetations of East Siang District of Arunachal Pradesh. 


\section{MATERIALS AND METHOD}

The present study was conducted in East Siang district of Arunachal Pradesh, it has unique characteristic vegetation types because of which it harbors different types of flora (Das 1986). The district is often known as the 'gateway to Arunachal Pradesh' and has derived its name from the mighty Siang River flowing through the area (Yumnam et al. 2011). It covers an area of $4005 \mathrm{~km}^{2}$, geographically located between $27^{\circ} 43^{\prime}$ to $29^{\circ} 20^{\prime} \mathrm{N}$ latitudes and $94^{\circ} 42^{\prime}$ to $95^{\circ} 35^{\prime}$ E longitudes at an elevation range of $130 \mathrm{~m}$ to $752 \mathrm{~m}$ a.m.s.l. The district experiences both tropical and subtropical climate. The annual rainfall of the district is recorded about $4168 \mathrm{~mm}$. East Siang district is predominantly inhabited by Adi community and they possess a strong base of traditional knowledge about forest structure and ecosystem functions. The study was conducted on the Tropical forests focusing mainly on bio-culturally important fruits.

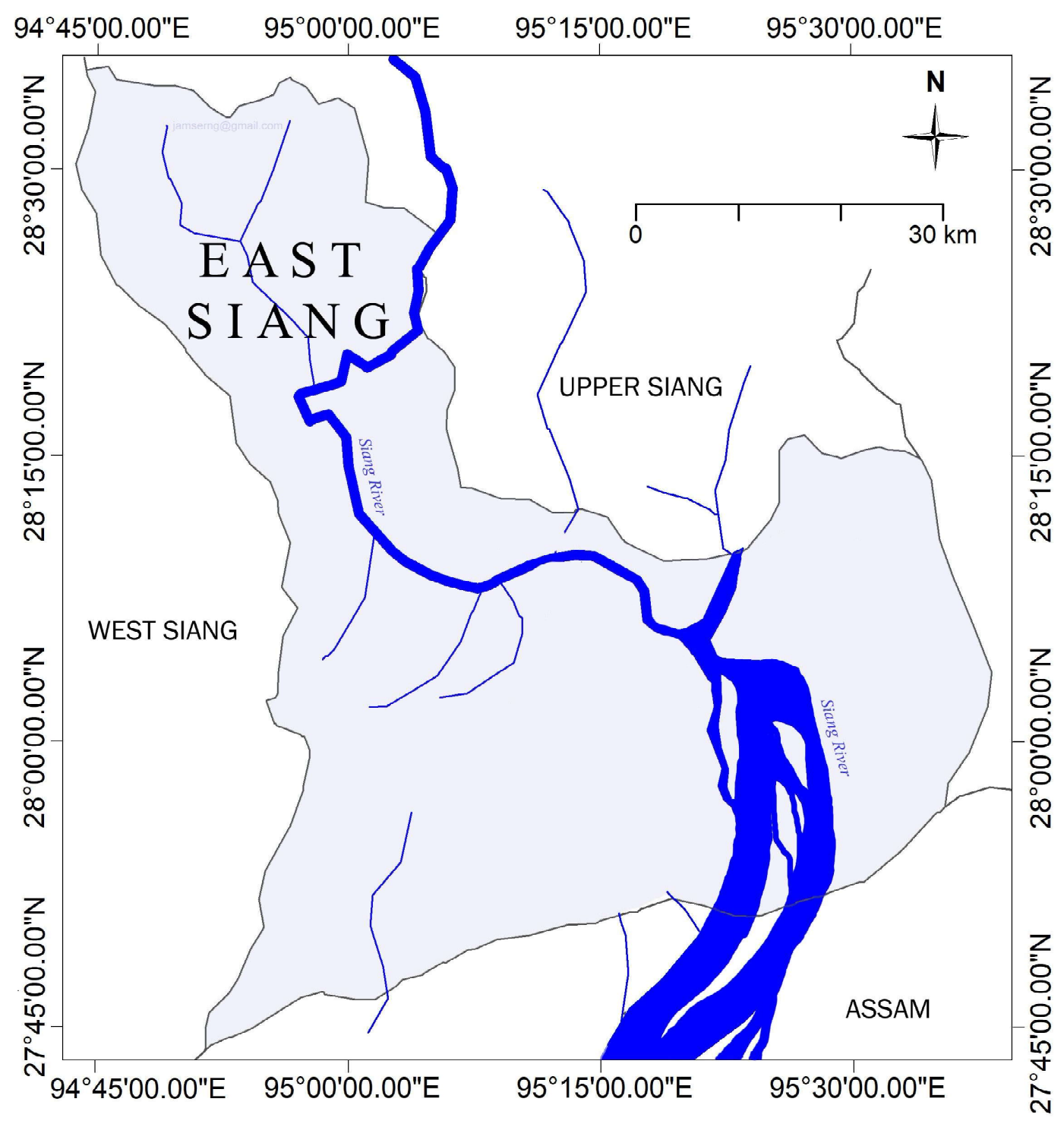

Map - 1. Study area, East Siang district of Arunachal Pradesh 
For the field survey, methods suggested by Martin (2008) was followed and was conducted in 8 villages viz. Mirsam, Mirbuk, Balek, Mebo, Ayeng, Bodak, Ledum and Rengging of East Siang District during the years 2017 - 2019. These villages are predominantly inhabited by the Adi Community. Prior Informed Consent (PIC) were taken from the potential informers like Traditional knowledge holders, farmers, priests, elders etc. of both the sexes and all the relevant ethnobotanical data were recorded in pre-structured questionnaire format and in field notebook. Voucher specimens were collected and processed following the methods as suggested by Jain \& Rao (1977). Plants were identified through consultation of standard regional floras such as Materials for the Flora of Arunachal Pradesh (Hajra et al. 1996; Giri et al.2008; Chowdhery et al.2009), Flora of Assam (Kanjilal et al. 1934 - 1940), The Flora of British India (Hooker, 1875 - 1897), eFlora of China, and e-Herbarium of Kew and the present status of nomenclature were taken from http:/ / www.worldfloraonline.org and www.plantsoftheworldonline.org. After the works are over the voucher specimens were deposited at HAU in the Department of Botany, Rajiv Gandhi University, Rono Hills, Doimukh, Arunachal Pradesh for future reference.

\section{RESULT AND DISCUSSION}

A total of 88 species from 56 genera, covering 39 families were found to be used by the Adi people of East Siang District. Of these, 87.5\% (77 spp.) are procured from wild and 12.5\% (11 spp.) are semi-domesticated (Table 1). Plants like Zanthoxylum rhetsa, Phoebe cooperiana, Litsea cubeba, Citrus latipes, Garcinia lanceifolia, Saurania punduana, etc. are partially domesticated by the local people though these are available naturally in the forest. This is an indication of the importance of these plants in their preferred diet as majority of these are edible. Now, fruits of these plants are also regularly marketed. Such incorporation of wild plants into the farming system will not only protect biodiversity but will also provide sufficient food and will contribute to the rural economy (Angami et al. 2006).

Considering the habit groups, tree was the most dominant with 48 species $(54.54 \%)$, followed by shrub (14 spp., $15.90 \%$ ), liana (6 spp., 6.81\%), geophytic herbs (5 spp., $5.68 \%$ ), annual herbs (4 spp., 4.54\%), shrubby climbers and perennial herbs had 2 species each (2 spp., $2.27 \%$ ). Whereas, herbaceous climber, stem parasite and suffrutescent plants had 1 species each (1 sp., $1.13 \%)$. So, at least for the edible fruits Adi people selected mainly arboreal or woody plants.

Considering the use categories, it was found that majority of the plants are food plants that consist of $77.1 \%$ (71spp.), followed by Hunting and fishing $9.8 \%$ (9 spp.), other uses $5.4 \%$ (5 spp.), medicinal $4.3 \%$ (4 spp.) and magico-religious beliefs and customs $3.3 \%$ (3 spp.).Arunachal Pradesh is the home to diverse range of fruits which forms important part of dietary supplement and at times used for curing many diseases and takes important part in their social culture and traditions since the time immemorial (Prakash et al. 2012; Singh \& Asha 2017). The present study in East Siang District reported more diversity of edible fruits than earlier studies conducted in Arunachal Pradesh (Singh \& Asha 2017; Lyndohet al. 2016). The study shows that a large number of wild fruits are used in the tropical regions, as due to their great diversity. Apart from food, many species are used as bait in traditional hunting and fishing techniques which are eaten by animals. Dependencies of present generation on domesticated crop plants, unavailability of sufficient written literatures and rapid modernization, the traditional knowledge in these societies are on the verge of extinction (Lungphi et al. 2018).

Moraceae was the most dominant family which comprises of $12.5 \%$ (11 spp.), followed by Rosaceae $9.1 \%$ (8 spp.), Zingiberaceae and Rutaceae with 5 species each (5.7\%), Arecaceae, 

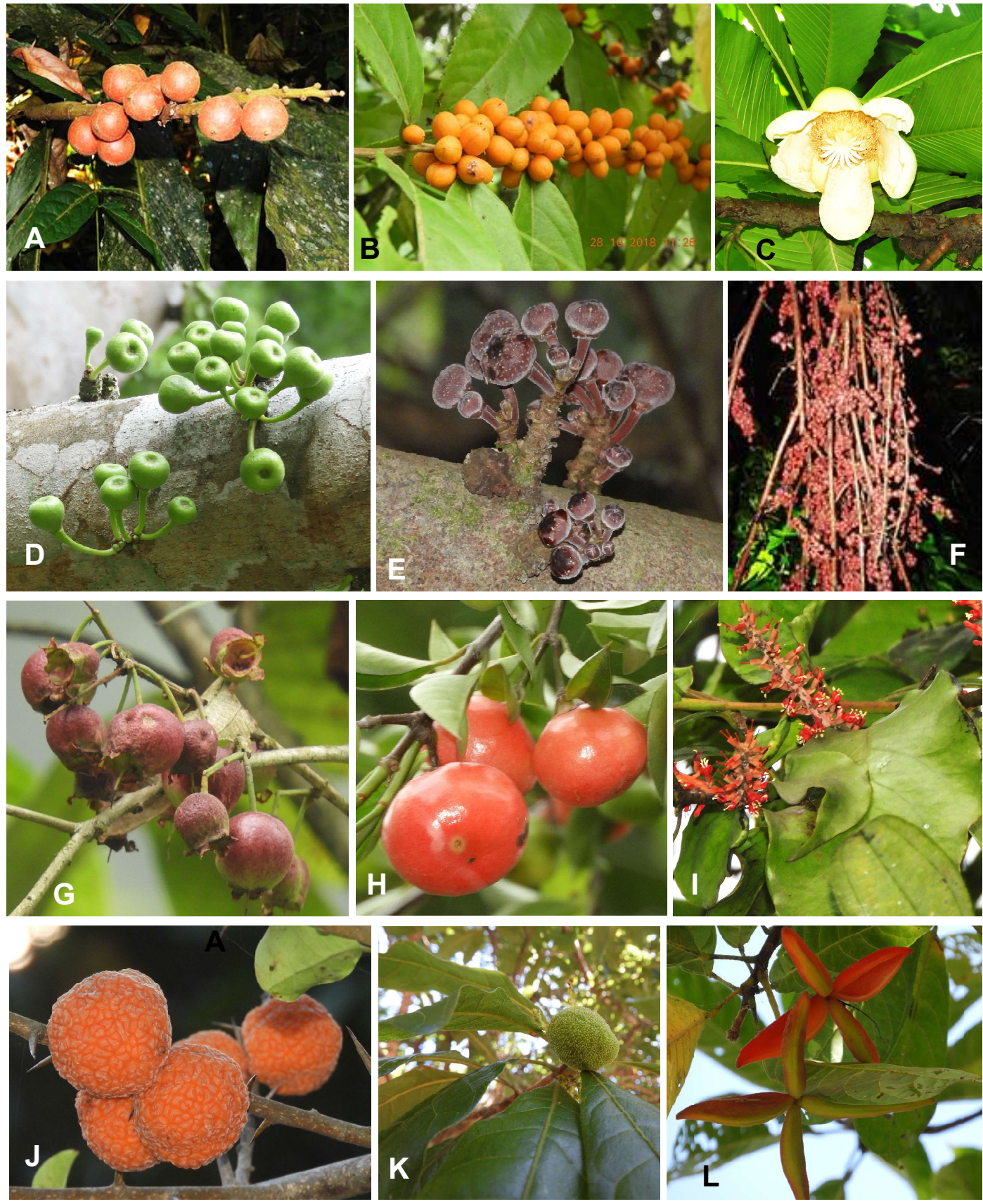

PLATE - I. Ethnobotanically useful fruits of Adi tribe: A. Alpinia roxburghï; B. Casearia vereca; C. Dillenia indica; D. Ficus variegata; E. Ficus oligodon; F. Ficus semicordata; G. Syayggium formosum; H. Garcinia lanceifolia; I. Helixanthera parasitica; J. Maclura cochinchinensis; K. Myrica esculenta; L. Sterculia lanceolata var. coccinea 
Table 1. Recorded ethnobotanical fruits used by the Adi tribe in the East Siang district of Arunachal Pradesh, India

[Abbreviations used: Habit: $\mathrm{HA}=$ Annual Herb; $\mathrm{HG}=$ Geophytic Herb; $\mathrm{HP}=$ Perennial Herb; $\mathrm{CH}=$ Herbaceous Climber; CS = Shrubby Climber; L = Liana; PR = Root Parasite; PS = Stem Parasite; $\mathrm{Sf}=$ Suffrutescent; $\mathrm{S}=$ Shrub; $\mathrm{T}=$ Tree. Uses: $\mathrm{F}=$ Food; $\mathrm{M}=$ Medicine; $\mathrm{HF}=$ Hunting and Fishing; $\mathrm{RBC}=$ Rituals, Beliefs and Customs; $\mathrm{O}=$ Others. IUCN status: $\mathrm{CR}=$ Critically Endangered; DD=Data Deficient; $\mathrm{NE}=$ Not Evaluated. Source: $\mathrm{WD}=$ Wild; $\mathrm{SM}=$ Semi-domesticated]

\begin{tabular}{|c|c|c|c|c|c|}
\hline $\begin{array}{l}\text { Botanical name [Family]; Voucher } \\
\text { specimen }\end{array}$ & Adi name & Habit & $\begin{array}{l}\text { Use and } \\
\text { application }\end{array}$ & $\begin{array}{l}\text { IUCN } \\
\text { Status }\end{array}$ & Source \\
\hline $\begin{array}{l}\text { Alpinia nigra (Gaertn.) Burtt } \\
\text { [Zingiberaceae]; MT3110 }\end{array}$ & $\begin{array}{l}\text { Gumba- } \\
\text { Bera }\end{array}$ & $\mathrm{HG}$ & $\begin{array}{l}\text { F: Ripe fruit eaten } \\
\text { raw }\end{array}$ & $\mathrm{NE}$ & WD \\
\hline $\begin{array}{l}\text { Alpinia roxburghii Sweet } \\
\text { [Zingiberaceae]; } M T-1683\end{array}$ & $\begin{array}{l}\text { Gumba- } \\
\text { Bera }\end{array}$ & HG & $\begin{array}{l}\text { F: Ripe fruit eaten } \\
\text { raw }\end{array}$ & $\mathrm{NE}$ & WD \\
\hline $\begin{array}{l}\text { Amomum maximum Roxb. } \\
\text { [Zingiberaceae]; MT-1591 }\end{array}$ & Taaling. & HG & $\begin{array}{l}\text { F: Seed eaten raw, } \\
\text { aromatic }\end{array}$ & $\mathrm{NE}$ & WD \\
\hline $\begin{array}{l}\text { Amomum subulatum Roxb. } \\
\text { [Zingiberaceae]; } M T-1592\end{array}$ & Taalingliite & HG & $\begin{array}{l}\text { F: Aromatic seeds } \\
\text { edible }\end{array}$ & DD & WD \\
\hline $\begin{array}{l}\text { Artocarpus lacucha Buch.Ham. } \\
\text { [Moraceae]; MT-1779 }\end{array}$ & Raami & $\mathrm{T}$ & $\begin{array}{l}\text { F: Taken raw when } \\
\text { ripe, sour }\end{array}$ & $\mathrm{NE}$ & WD \\
\hline $\begin{array}{l}\text { Baccaurea ramiflora Lour. } \\
\text { [Phyllanthaceae]; } M T-1629\end{array}$ & $\begin{array}{l}\text { Bureng, } \\
\text { Buri }\end{array}$ & $\mathrm{T}$ & $\begin{array}{l}\text { F:Aril on seeds } \\
\text { edible }\end{array}$ & $\mathrm{NE}$ & WD \\
\hline $\begin{array}{l}\text { Beaumontia grandiflora Wall. } \\
\text { [Apocynaceae]; } M T-1636\end{array}$ & $\begin{array}{l}\text { Dongko- } \\
\text { Riyo }\end{array}$ & $\mathrm{L}$ & $\begin{array}{l}\text { RBC: seed coma } \\
\text { used to decorate } \\
\text { traditional hats } \\
\text { "Leebro" - worn } \\
\text { during war dance } \\
\text { (Taapu) }\end{array}$ & $\mathrm{NE}$ & WD \\
\hline $\begin{array}{l}\text { Bombax ceiba L. [Malvaceae]; MT- } \\
1699\end{array}$ & $\begin{array}{l}\text { HingyoGyo } \\
\text { mur, Simul }\end{array}$ & $\mathrm{T}$ & $\begin{array}{l}\text { O: Seed floss as } \\
\text { stuffing material for } \\
\text { pillows }\end{array}$ & $\mathrm{NE}$ & WD \\
\hline $\begin{array}{l}\text { Calamus erectus Roxb. [Arecaceae]; } \\
\text { MT-1562 }\end{array}$ & Tara & $\mathrm{L}$ & F: fruits sour & $\mathrm{NE}$ & $\mathrm{SM}$ \\
\hline $\begin{array}{l}\text { Calamus flagellum Griff. ex Mart. } \\
\text { [Arecaceae]; MT-1541 }\end{array}$ & Yoyi, Jeying & $\mathrm{L}$ & F: Ripe fruits edible & $\mathrm{NE}$ & SM \\
\hline $\begin{array}{l}\text { Canarium strictum Roxb. } \\
\text { [Burseraceae]; } M T-1687\end{array}$ & $\begin{array}{l}\text { Hilum, } \\
\text { Komkel }\end{array}$ & $\mathrm{T}$ & F: Fruits edible & $\mathrm{NE}$ & WD \\
\hline $\begin{array}{l}\text { Casearia vareca Roxb. [Salicaceae]; } \\
M T-1597\end{array}$ & Sipe-Sïle & $\mathrm{S}$ & $\begin{array}{l}\text { HF: Ripe ones used } \\
\text { as bait in traditional } \\
\text { trap (Etku) to hunt } \\
\text { birds and rodents }\end{array}$ & $\mathrm{NE}$ & WD \\
\hline $\begin{array}{l}\text { Castanopsis indica (Roxb. ex Lindl.) } \\
\text { A.DC. [Fagaceae]; MT-1602 }\end{array}$ & Sïrang & $\mathrm{T}$ & $\begin{array}{l}\text { F: Endosperm eaten } \\
\text { raw or roasted }\end{array}$ & $\mathrm{NE}$ & WD \\
\hline $\begin{array}{l}\text { Castanopsis purpurella (Miq.) } \\
\text { N.P.Balakr. [Fagaceae]; MT-1618 }\end{array}$ & Angke & $\mathrm{T}$ & $\begin{array}{l}\text { F: Endosperm eaten } \\
\text { raw or roasted }\end{array}$ & $\mathrm{NE}$ & WD \\
\hline $\begin{array}{l}\text { Choerospondias axillaris (Roxb.) } \\
\text { B.L. Burtt\&A.W.Hill [Anacardiaceae]; } \\
\text { MT-1529 }\end{array}$ & Belam & $\mathrm{T}$ & $\begin{array}{l}\text { F: Ripe fruits sweet } \\
\text { and edible } \\
\text { HF: bait for hunting } \\
\text { deer }\end{array}$ & $\mathrm{NE}$ & WD \\
\hline
\end{tabular}




\begin{tabular}{|c|c|c|c|c|c|}
\hline $\begin{array}{l}\text { Botanical name [Family]; Voucher } \\
\text { specimen }\end{array}$ & Adi name & Habit & $\begin{array}{l}\text { Use and } \\
\text { application }\end{array}$ & $\begin{array}{l}\text { IUCN } \\
\text { Status }\end{array}$ & Source \\
\hline $\begin{array}{l}\text { Cinnamomum bejolghota (Buch.- } \\
\text { Ham.) Sweet [Lauraceae]; MT-1690 }\end{array}$ & Hipir-Ayin & $\mathrm{T}$ & $\begin{array}{l}\text { F: Young fruits } \\
\text { steamed as chutney }\end{array}$ & $\mathrm{NE}$ & WD \\
\hline $\begin{array}{l}\text { Citrus indica Yu. Tanaka [Rutaceae]; } \\
M T-1681\end{array}$ & $\begin{array}{l}\text { Goyeng- } \\
\text { Hingkiin }\end{array}$ & $\mathrm{T}$ & $\begin{array}{l}\text { F: Ripe fruits sour, } \\
\text { taken raw }\end{array}$ & $\mathrm{NE}$ & WD \\
\hline $\begin{array}{l}\text { Citrus latipes (Swingle) Yu.Tanaka } \\
\text { [Rutaceae]; MT-1809 }\end{array}$ & $\begin{array}{l}\text { Hinnong/ } \\
\text { Hingkom }\end{array}$ & $\mathrm{T}$ & $\begin{array}{l}\text { F: Fruit taken raw, } \\
\text { sour }\end{array}$ & $\mathrm{NE}$ & SM \\
\hline $\begin{array}{l}\text { Citrus medica L. [ Rutaceae]; } M T \text { - } \\
1688\end{array}$ & Hingkom & $\mathrm{S}$ & $\begin{array}{l}\text { F: Fruit taken raw, } \\
\text { sour }\end{array}$ & $\mathrm{NE}$ & WD \\
\hline $\begin{array}{l}\text { Coffea benghalensis B.Heyne ex } \\
\text { Schult.[Rubiaceae] MT-6002 }\end{array}$ & - & $\mathrm{S}$ & $\begin{array}{l}\text { F: Ripe fruit eaten } \\
\text { raw by children }\end{array}$ & $\mathrm{NE}$ & WD \\
\hline $\begin{array}{l}\text { Cordia dichotoma G.Forst } \\
\text { [Boraginaceae]; } M T-1514\end{array}$ & Jongge & $\mathrm{T}$ & $\begin{array}{l}\text { O: Sticky mesocarp } \\
\text { as glue for light } \\
\text { materials like paper }\end{array}$ & $\mathrm{NE}$ & WD \\
\hline $\begin{array}{l}\text { Curculigo capitulata (Lour.) Kuntze } \\
\text { [Hypoxidaceae]; } M T-1548\end{array}$ & Tayek. & $\mathrm{HP}$ & F: Fruit edible & $\mathrm{NE}$ & WD \\
\hline $\begin{array}{l}\text { Curculigo prainiana (Deb) Bennet } \\
\text { \& Raizada Hypoxidaceae]; MT-1815 }\end{array}$ & Tayek & $\mathrm{PH}$ & F: Fruit edible & $\mathrm{NE}$ & WD \\
\hline $\begin{array}{l}\text { Dillenia indica L. [Dilleniaceae]; } \\
M T-1593\end{array}$ & Sompa & $\mathrm{T}$ & $\begin{array}{l}\text { F: Acrescent calyx } \\
\text { eaten raw }\end{array}$ & $\mathrm{NE}$ & WD \\
\hline $\begin{array}{l}\text { Duchesnea indica (Jacks.) Focke } \\
\text { [Rosaceae]; } M T-1648\end{array}$ & $\begin{array}{l}\text { Eki- } \\
\text { Tangkin }\end{array}$ & $\mathrm{HA}$ & $\begin{array}{l}\text { F: Ripe fruit eaten } \\
\text { raw, watery }\end{array}$ & $\mathrm{NE}$ & WD \\
\hline $\begin{array}{l}\text { Ficus auriculata Lour. [Moraceae]; } \\
M T-1756\end{array}$ & $\begin{array}{l}\text { Paapop, } \\
\text { Tapok }\end{array}$ & $\mathrm{T}$ & F: Ripe hypanthodia & $\mathrm{NE}$ & WD \\
\hline $\begin{array}{l}\text { Ficus fulva Reinw. ex Blume } \\
\text { [Moraceae];MT-6004 }\end{array}$ & Takpi & $\mathrm{T}$ & $\begin{array}{l}\text { O: Hypanthodia as } \\
\text { fodder for cattle }\end{array}$ & $\mathrm{NE}$ & WD \\
\hline $\begin{array}{l}\text { Ficus geocarpa Teijsm. ex. Miq. } \\
\text { [Moraceae]; } M T-1641\end{array}$ & EeBerii & $\mathrm{T}$ & $\begin{array}{l}\text { F:Ripe hypanthodia } \\
\text { edible, sweet }\end{array}$ & $\mathrm{NE}$ & WD \\
\hline $\begin{array}{l}\text { Ficus heteropleura Blume } \\
\text { [Moraceae]; MT-1790 }\end{array}$ & - & $\mathrm{T}$ & $\begin{array}{l}\text { HF: Ripe } \\
\text { hypanthodia used as } \\
\text { bait for birds }\end{array}$ & $\mathrm{NE}$ & WD \\
\hline $\begin{array}{l}\text { Ficus hispida L.f. [Moraceae]; } \\
\text { MT-6003 }\end{array}$ & $\begin{array}{l}\text { Ek-itakuk; } \\
\text { Tapang; } \\
\text { Eki-tapang }\end{array}$ & $\mathrm{T}$ & $\begin{array}{l}\text { O: Hypanthodia as } \\
\text { fodder for cattle }\end{array}$ & $\mathrm{NE}$ & WD \\
\hline $\begin{array}{l}\text { Ficus oligodon Miq. [Moraceae]; } \\
M T-1761\end{array}$ & $\begin{array}{l}\text { Pameng; } \\
\text { Tapang }\end{array}$ & $\mathrm{T}$ & $\begin{array}{l}\text { F: Ripe hypanthodia } \\
\text { edible, sweet }\end{array}$ & $\mathrm{NE}$ & WD \\
\hline $\begin{array}{l}\text { Ficus semicordata Buch. Ham. ex } \\
\text { Sm. [Moraceae]; } M T-1574\end{array}$ & Takuk & $\mathrm{T}$ & $\begin{array}{l}\text { F: Ripe hypanthodia } \\
\text { edible, sweet }\end{array}$ & $\mathrm{NE}$ & WD \\
\hline $\begin{array}{l}\text { Ficus variegata Blume [Moraceae]; } \\
M T-1588\end{array}$ & Taasik & $\mathrm{T}$ & $\begin{array}{l}\text { F: Ripe hypanthodia } \\
\text { edible, sweet }\end{array}$ & $\mathrm{NE}$ & WD \\
\hline $\begin{array}{l}\text { Fissistigma bicolor (Roxb.) Merr. } \\
\text { [Annonaceae]; } M T-1816\end{array}$ & Rika-Riya & $\bar{L}$ & $\begin{array}{l}\text { F: Ripe fruits sweet, } \\
\text { taken raw }\end{array}$ & $\mathrm{NE}$ & WD \\
\hline $\begin{array}{l}\text { Fissistigma polyanthum (Hook.f. \& } \\
\text { Thomson) Merr. [Annonaceae]; MT- } \\
1772\end{array}$ & Rika-Riya & $\mathrm{S}$ & $\begin{array}{l}\text { F: Eaten ripe one } \\
\text { raw, sweet }\end{array}$ & $\mathrm{NE}$ & WD \\
\hline
\end{tabular}


Momang Taram et al. 103

\begin{tabular}{|c|c|c|c|c|c|}
\hline $\begin{array}{l}\text { Botanical name [Family]; Voucher } \\
\text { specimen }\end{array}$ & Adi name & Habit & $\begin{array}{l}\text { Use and } \\
\text { application }\end{array}$ & $\begin{array}{l}\text { IUCN } \\
\text { Status }\end{array}$ & Source \\
\hline $\begin{array}{l}\text { Garcinia anomala Planch. \&Triana } \\
\text { [Clusiaceae]; MT-1561 }\end{array}$ & Taraak & $\mathrm{T}$ & F: Fruit edible, sour & $\mathrm{NE}$ & WD \\
\hline $\begin{array}{l}\text { Garcinia lanceifolia Roxb. } \\
\text { [Clusiaceae]; } M T-1560\end{array}$ & Taraak & $\mathrm{T}$ & F:Fruit edible, sour & $\mathrm{NE}$ & SM \\
\hline $\begin{array}{l}\text { Garcinia pedunculata Roxb. } \\
\text { exBuch. Ham. [Clusiaceae]; MT-1586 }\end{array}$ & Tabing & $\mathrm{T}$ & $\begin{array}{l}\text { F: Ripe ones eaten } \\
\text { raw, sweet; M: } \\
\text { Smoked fruit-wall } \\
\text { taken orally to treat } \\
\text { gastrointestinal } \\
\text { problems }\end{array}$ & $\mathrm{NE}$ & WD \\
\hline $\begin{array}{l}\text { Gynocardia odorata R.Br. } \\
\text { [Achariaceae]; } M T-1731\end{array}$ & $\begin{array}{l}\text { Mondo- } \\
\text { Tulpi, } \\
\text { Sibetulpi }\end{array}$ & $\mathrm{T}$ & $\begin{array}{l}\text { HF: Fruit paste } \\
\text { mixed in water for } \\
\text { stupefying fishes }\end{array}$ & $\mathrm{NE}$ & WD \\
\hline $\begin{array}{l}\text { Helixanthera parasitica Lour. } \\
\text { [Loranthaceae]; MT-1555 }\end{array}$ & Tasik & PS & $\begin{array}{l}\text { F: Ripe fruit eaten } \\
\text { raw, sweet }\end{array}$ & $\mathrm{NE}$ & WD \\
\hline $\begin{array}{l}\text { Heteropanax fragrans (Roxb.) } \\
\text { Seem. [Araliaceae]; MT-1656 }\end{array}$ & $\begin{array}{l}\text { Gaatum- } \\
\text { Bopang }\end{array}$ & $\mathrm{T}$ & $\begin{array}{l}\text { HF: Fruit as bait to } \\
\text { trap rodents and } \\
\text { birds }\end{array}$ & $\mathrm{NE}$ & WD \\
\hline $\begin{array}{l}\text { Hodgsonia macrocarpa (Blume) } \\
\text { Cong. [Cucurbitaceae]; MT-1552 }\end{array}$ & Tatar-Api & $\mathrm{L}$ & $\begin{array}{l}\text { F: Embryo edible } \\
\text { after cooking }\end{array}$ & $\mathrm{NE}$ & WD \\
\hline $\begin{array}{l}\text { Hornstedtia arunachalensis S. } \\
\text { Triphathi \& V.Prakash } \\
\text { [Zingiberaceae]; } M T-1627\end{array}$ & Bele-Belaak & HG & F: fruits eaten raw & $\mathrm{NE}$ & WD \\
\hline $\begin{array}{l}\text { Litsea cubeba (Lour.) Pers. } \\
\text { [Lauraceae]; MT-1777 }\end{array}$ & Rayil, Tayir & $\mathrm{T}$ & $\begin{array}{l}\text { F: Fruit as } \\
\text { condiment, strongly } \\
\text { aromatic }\end{array}$ & $\mathrm{NE}$ & SM \\
\hline $\begin{array}{l}\text { Livistona jenkinsiana Griff. } \\
\text { [Arecaceae]; } M T-1520\end{array}$ & Taek & $\mathrm{T}$ & F: Ripe fruits edible & $\mathrm{NE}$ & SM \\
\hline $\begin{array}{l}\text { Macaranga peltata (Roxb.) } \\
\text { Mull.Arg. [Euphorbiaceae]; MT-1521 }\end{array}$ & Lagar & $\mathrm{T}$ & $\begin{array}{l}\text { HF: Fruit as bait for } \\
\text { birds and rodents }\end{array}$ & $\mathrm{NE}$ & WD \\
\hline $\begin{array}{l}\text { Maclura cochinchinensis (Lour.) } \\
\text { Corner [Moraceae]; MT-1808 }\end{array}$ & $\begin{array}{l}\text { Tanyum- } \\
\text { Tang }\end{array}$ & S & $\begin{array}{l}\text { F: Ripe fruit eaten, } \\
\text { sweet }\end{array}$ & $\mathrm{NE}$ & WD \\
\hline $\begin{array}{l}\text { Maesa indica (Roxb.) A.DC. } \\
{[\text { Primulaceae }] ; M T-1654}\end{array}$ & $\begin{array}{l}\text { Etjun- } \\
\text { Jayun, } \\
\text { Nyanyur }\end{array}$ & $\mathrm{S}$ & $\begin{array}{l}\text { F: Ripe fruits eaten } \\
\text { raw }\end{array}$ & $\mathrm{NE}$ & WD \\
\hline $\begin{array}{l}\text { Mangifera sylvatica Roxb. } \\
\text { [Anacardiaceae]; MT-1686 }\end{array}$ & $\begin{array}{l}\text { Hidum- } \\
\text { Tagung, } \\
\text { Nyomrang- } \\
\text { tagung }\end{array}$ & $\mathrm{T}$ & $\begin{array}{l}\text { F: Ripe fruits edible, } \\
\text { sour }\end{array}$ & $\mathrm{NE}$ & WD \\
\hline $\begin{array}{l}\text { Melastoma malabathricum L. } \\
\text { [Melastomataceae]; MT-1709 }\end{array}$ & $\begin{array}{l}\text { Kasii-Rai, } \\
\text { Jojer }\end{array}$ & Sf & F: Fruits eaten raw & $\mathrm{NE}$ & WD \\
\hline $\begin{array}{l}\text { Melothria trilobata Cogn. } \\
\text { [Cucurbitaceae]; MT-1635 }\end{array}$ & $\begin{array}{l}\text { Dongkong- } \\
\text { kayong }\end{array}$ & $\mathrm{CH}$ & $\begin{array}{l}\text { F: Ripe fruits eaten } \\
\text { raw }\end{array}$ & $\mathrm{NE}$ & WD \\
\hline $\begin{array}{l}\text { Microtropis discolor (Wall.) Arn. } \\
\text { [Celastraceae]; MT-1827 }\end{array}$ & - & $\mathrm{T}$ & $\begin{array}{l}\text { HF: Red seed used } \\
\text { as bait for rodents }\end{array}$ & $\mathrm{NE}$ & WD \\
\hline
\end{tabular}


104 Ethnic uses of wild fruits by Adi tribe

\begin{tabular}{|c|c|c|c|c|c|}
\hline $\begin{array}{l}\text { Botanical name [Family]; Voucher } \\
\text { specimen }\end{array}$ & Adi name & Habit & $\begin{array}{l}\text { Use and } \\
\text { application }\end{array}$ & $\begin{array}{l}\text { IUCN } \\
\text { Status }\end{array}$ & Source \\
\hline Morus alba L. [Moraceae]; MT-1789 & Nini-guti & $\mathrm{T}$ & $\begin{array}{l}\text { F: Ripe ones sweet, } \\
\text { edible }\end{array}$ & $\mathrm{NE}$ & SM \\
\hline $\begin{array}{l}\text { Musa aurantiaca G.Mann ex Baker } \\
\text { [Musaceae]; MT-1640 }\end{array}$ & Dumji & $\mathrm{HP}$ & F: Ripe fruit sweet & $\mathrm{NE}$ & WD \\
\hline $\begin{array}{l}\text { Myrica esculenta Buch.Ham. ex } \\
\text { D.Don [Myricaceae]; MT-1551 }\end{array}$ & Tatir & $\mathrm{T}$ & $\begin{array}{l}\text { F: Ripe ones eaten } \\
\text { raw }\end{array}$ & $\mathrm{NE}$ & WD \\
\hline $\begin{array}{l}\text { Nephelium lappaceum L. } \\
\text { [Sapindaceae]; MT-1583 }\end{array}$ & Tadar & $\mathrm{T}$ & $\begin{array}{l}\text { F: Ripe ones eaten } \\
\text { raw, sour and sweet }\end{array}$ & $\mathrm{NE}$ & WD \\
\hline $\begin{array}{l}\text { Pandanus furcatus Roxb. } \\
\text { [Pandanaceae]; } M T-1788\end{array}$ & Tako & $\mathrm{T}$ & F: Dried seed edible & $\mathrm{NE}$ & WD \\
\hline $\begin{array}{l}\text { Persicaria capitate (Buch.Ham. ex } \\
\text { D.Don) H.Gross [Polygonaceae]; MT- } \\
1624\end{array}$ & $\begin{array}{l}\text { Babing- } \\
\text { kaling, } \\
\text { Mijingkalin } \\
g\end{array}$ & HA & $\begin{array}{l}\text { F: Ripe fruit eaten } \\
\text { raw }\end{array}$ & $\mathrm{NE}$ & WD \\
\hline $\begin{array}{l}\text { Phoebe cooperiana P.C. Kanjilal \& } \\
\text { Das [Lauraceae]; } M T-1563\end{array}$ & Tapir & $\mathrm{T}$ & $\begin{array}{l}\text { F: Ripe fruits eaten } \\
\text { raw }\end{array}$ & $\mathrm{NE}$ & SM \\
\hline $\begin{array}{l}\text { Phrynium pubinerve Blume } \\
\text { [Marantaceae]; MT-1649 }\end{array}$ & Ekekam & $\mathrm{HP}$ & F: Seeds eaten raw & $\mathrm{NE}$ & SM \\
\hline $\begin{array}{l}\text { Physalis lagascae Roem. \&Schult. } \\
\text { [Solanaceae]; MT-1702 }\end{array}$ & Jojing-belang & HA & $\begin{array}{l}\text { F: Ripe ones eaten } \\
\text { raw }\end{array}$ & $\mathrm{NE}$ & WD \\
\hline $\begin{array}{l}\text { Rhaphidophora decursiva (Roxb.) } \\
\text { Schott [Araceae]; MT-6001 }\end{array}$ & Talo & $\mathrm{PH}$ & $\begin{array}{l}\text { HF: Fruit used as } \\
\text { bait for fish }\end{array}$ & $\mathrm{NE}$ & WD \\
\hline $\begin{array}{l}\text { Rhus chinensis Mill. } \\
\text { [Anacardiaceae]; MT-1580 }\end{array}$ & Tagmo & $\mathrm{T}$ & $\begin{array}{l}\text { M: Cooked with } \\
\text { wild mushroom to } \\
\text { avoid food } \\
\text { poisoning }\end{array}$ & $\mathrm{NE}$ & WD \\
\hline $\begin{array}{l}\text { Rhynchotechum vestitum Wall. ex } \\
\text { C.B. Clarke [Gesneriaceae]; MT-1706 }\end{array}$ & Jongkot & $\mathrm{S}$ & $\begin{array}{l}\text { F: Fruits watery, } \\
\text { eaten raw }\end{array}$ & $\mathrm{NE}$ & WD \\
\hline $\begin{array}{l}\text { Rubus alceifolius Poir. [Rosaceae]; } \\
M T-1763\end{array}$ & Pasi-Payi & $\mathrm{S}$ & $\begin{array}{l}\text { F: Ripe ones eaten } \\
\text { raw, sweet }\end{array}$ & $\mathrm{NE}$ & WD \\
\hline $\begin{array}{l}\text { Rubus ellipticus Sm. [Rosaceae]; } \\
M T-1759\end{array}$ & $\begin{array}{l}\text { Pakkom- } \\
\text { Tayin }\end{array}$ & $\mathrm{S}$ & $\begin{array}{l}\text { F: Ripe oned eaten } \\
\text { raw, sweet }\end{array}$ & $\mathrm{NE}$ & WD \\
\hline $\begin{array}{l}\text { Rubus niveus Thumb. [Rosaceae]; } \\
M T-1542\end{array}$ & $\begin{array}{l}\text { Yokpo- } \\
\text { Pongkung }\end{array}$ & CS & $\begin{array}{l}\text { F: Ripe ones eaten } \\
\text { raw, sweet }\end{array}$ & $\mathrm{NE}$ & WD \\
\hline $\begin{array}{l}\text { Rubus paniculatus Sm. [Rosaceae]; } \\
\text { MT-1569 }\end{array}$ & Tangkin & CS & $\begin{array}{l}\text { F: Ripe ones eaten } \\
\text { raw, sweet }\end{array}$ & $\mathrm{NE}$ & WD \\
\hline $\begin{array}{l}\text { Rubus rosifolius Sm. [Rosaceae]; } \\
\text { MT-1831 }\end{array}$ & Tangkin & $\mathrm{S}$ & $\begin{array}{l}\text { F: Ripe ones eaten } \\
\text { raw }\end{array}$ & $\mathrm{NE}$ & WD \\
\hline $\begin{array}{l}\text { Rubus sieboldii Blume [R. moluccanus } \\
\text { L.], [Rosaceae]; MT-1567 }\end{array}$ & $\begin{array}{l}\text { Tapa- } \\
\text { Tara }\end{array}$ & $\mathrm{S}$ & $\begin{array}{l}\text { F: Ripe fruits eaten } \\
\text { raw, sweet }\end{array}$ & $\mathrm{NE}$ & WD \\
\hline $\begin{array}{l}\text { Rubussumatranus Miq. [Rosaceae]; } \\
\text { MT-1713 }\end{array}$ & $\begin{array}{l}\text { kinbu- } \\
\text { Beru }\end{array}$ & CS & $\begin{array}{l}\text { F: Ripe fruits edible, } \\
\text { sweet }\end{array}$ & $\mathrm{NE}$ & WD \\
\hline $\begin{array}{l}\text { Sapindus mukorossi Gaertn. } \\
\text { [Sapindaceae]; MT-6006 }\end{array}$ & $\begin{array}{l}\text { Kuku- } \\
\text { rabak }\end{array}$ & $\mathrm{T}$ & $\begin{array}{l}\text { O: Dried fruit used } \\
\text { to clean ornaments }\end{array}$ & $\mathrm{NE}$ & WD \\
\hline $\begin{array}{l}\text { Saurauia armata Kurtz } \\
\text { [Actinidiaceae]; MT-1619 }\end{array}$ & Anpum & $\mathrm{T}$ & $\begin{array}{l}\text { F: Ripe fruits eaten } \\
\text { raw, sweet }\end{array}$ & $\mathrm{NE}$ & WD \\
\hline
\end{tabular}




\begin{tabular}{|c|c|c|c|c|c|}
\hline $\begin{array}{l}\text { Botanical name [Family]; Voucher } \\
\text { specimen }\end{array}$ & Adi name & Habit & $\begin{array}{l}\text { Use and } \\
\text { application }\end{array}$ & $\begin{array}{l}\text { IUCN } \\
\text { Status }\end{array}$ & Source \\
\hline $\begin{array}{l}\text { Saurauia griffithii Dyer } \\
\text { [Actinidiaceae]; MT-1829 }\end{array}$ & Taan & $\mathrm{T}$ & $\begin{array}{l}\text { F: Ripe ones eaten } \\
\text { raw }\end{array}$ & $\mathrm{NE}$ & WD \\
\hline $\begin{array}{l}\text { Saurauia napaulensis DC. } \\
\text { [Actinidiaceae]; } M T-1590\end{array}$ & Taan & $\mathrm{T}$ & $\begin{array}{l}\text { F: Ripe fruits eaten } \\
\text { raw, sweet }\end{array}$ & $\mathrm{NE}$ & WD \\
\hline $\begin{array}{l}\text { Saurauia punduana Wall. } \\
\text { [Actinidiaceae]; MT-1589 }\end{array}$ & Taan & $\mathrm{T}$ & $\begin{array}{l}\text { F: Ripe fruits eaten } \\
\text { raw, sweet }\end{array}$ & CR & SM \\
\hline $\begin{array}{l}\text { Solanum torvum Sw. [Solanaceae]; } \\
M T-1526\end{array}$ & $\begin{array}{l}\text { Kodul } \\
\text { Migom } \\
\text { Kopi/Kopi- } \\
\text { Piite }\end{array}$ & $\mathrm{S}$ & $\begin{array}{l}\text { F: Young ones, } \\
\text { bitter, cooked as } \\
\text { chutney }\end{array}$ & $\mathrm{NE}$ & WD \\
\hline $\begin{array}{l}\text { Solanum viarum Dunal [Solanaceae]; } \\
M T-1525\end{array}$ & $\begin{array}{l}\text { Peeli-Taang, } \\
\text { Kili-taang }\end{array}$ & HA & $\begin{array}{l}\text { M: Warmed on fire } \\
\text { and then applied on } \\
\text { infected teeth }\end{array}$ & $\mathrm{NE}$ & WD \\
\hline $\begin{array}{l}\text { Spondias pinnata (L.f.) Kurz } \\
\text { [Anacardiaceae]; } M T-1530\end{array}$ & $\begin{array}{l}\text { Dorgu- } \\
\text { dorge, Dorge }\end{array}$ & $\mathrm{T}$ & F: Eaten raw, sour & $\mathrm{NE}$ & WD \\
\hline $\begin{array}{l}\text { Sterculia lanceolata var. coccinea } \\
\text { (Jack) Phengklai[Malvaceae]; } M T-1549\end{array}$ & Tayam & $\mathrm{T}$ & $\begin{array}{l}\text { F: Immature seeds } \\
\text { eaten raw and } \\
\text { mature ones roasted; } \\
\text { RBC: open ripe } \\
\text { fruit is hung on the } \\
\text { door to scare the } \\
\text { evil spirits }\end{array}$ & $\mathrm{NE}$ & WD \\
\hline $\begin{array}{l}\text { Sterculia striatiflora Mast. } \\
{[\text { Malvaceae]; MT-1786 }}\end{array}$ & Tayam & $S$ & $\begin{array}{l}\text { F: Immature seeds } \\
\text { eaten raw and } \\
\text { mature ones roasted; } \\
\text { RBC: open ripe } \\
\text { fruit is hung on the } \\
\text { door to scare the } \\
\text { evil spirits }\end{array}$ & $\mathrm{NE}$ & WD \\
\hline $\begin{array}{l}\text { Stixis suaveolens (Roxb.) Pierre } \\
\text { [Capparaceae]; MT-1613 }\end{array}$ & $\begin{array}{l}\text { Rokpo- } \\
\text { ketum - } \\
\text { kelum }\end{array}$ & $\mathrm{L}$ & $\begin{array}{l}\text { F: Ripe ones taken } \\
\text { raw, sweet }\end{array}$ & $\mathrm{NE}$ & WD \\
\hline $\begin{array}{l}\text { Syzygium formosum (Wall.) Masam } \\
\text { [Myrtaceae]; MT-1826 }\end{array}$ & Ponkan & $\mathrm{T}$ & $\begin{array}{l}\text { F: Ripe ones eaten } \\
\text { raw }\end{array}$ & $\mathrm{NE}$ & WD \\
\hline $\begin{array}{l}\text { Syzygium fruticosum DC. } \\
\text { [Myrtaceae]; } M T-1828\end{array}$ & Jongkeng & $\mathrm{T}$ & $\begin{array}{l}\text { F: Ripe ones eaten } \\
\text { raw }\end{array}$ & $\mathrm{NE}$ & WD \\
\hline $\begin{array}{l}\text { Terminalia chebula Retz. } \\
\text { [Combretaceae]; MT-6000 }\end{array}$ & Ilikang & $\mathrm{T}$ & $\begin{array}{l}\text { M: Fruit taken } \\
\text { orally for cold and } \\
\text { cough }\end{array}$ & $\mathrm{NE}$ & WD \\
\hline $\begin{array}{l}\text { Trevesia palmata (Roxb. ex Lindl.) } \\
\text { Vis. [Araliaceae]; MT-1679 }\end{array}$ & $\begin{array}{l}\text { Gorpak, } \\
\text { Tagor }\end{array}$ & $\mathrm{T}$ & $\begin{array}{l}\text { F: Young fruits } \\
\text { bitter and cooked as } \\
\text { chutney }\end{array}$ & $\mathrm{NE}$ & WD \\
\hline $\begin{array}{l}\text { Zanthoxylum armatum DC. } \\
\text { [Rutaceae]; } M T-1535\end{array}$ & $\begin{array}{l}\text { Ombeng, } \\
\text { Ombe }\end{array}$ & $\mathrm{S}$ & F: Fruits as spice & $\mathrm{NE}$ & WD \\
\hline $\begin{array}{l}\text { Zanthoxylum rhetsa (Roxb.) DC. } \\
\text { [Rutaceae]; MT-1533 }\end{array}$ & Onger & $\mathrm{T}$ & $\begin{array}{l}\text { HF: Fruits paste } \\
\text { used as stupefying } \\
\text { agents for fish } \\
\text { poisoning }\end{array}$ & $\mathrm{NE}$ & SM \\
\hline
\end{tabular}


Clusiaceae and Lauraceae with 3 species each (3.40 \%). Among the genera Ficus and Rubus were the most dominant (7 species each), followed by Saurania (4 species), Garcinia and Citrus (3 species each).According to IUCN Red list categories (IUCN 2020)Saurania punduanais evaluated as Critically Endangered (CR), Amomum subulatum as Data deficient (DD) and remaining species (86 spp., $97.72 \%$ ) are not evaluated.

Arunachal Pradesh is facing several threats and biotic pressures in the form of shifting cultivation, grazing, forest fires, loss of pollinators, commercial monoculture plantations and illegal extraction of forest products and diversion of forest land for developmental projects (Kumar \& Chaudhry 2015; Kanwal \& Lodhi 2018). Because of this many species of plants are now on the verge of extinction. Although most of the species in the study area are not listed in IUCN categories, they may abundantly distributed globally but species viz. Saurania griffithii, Citrus latipes, Citrus indica, Ficus geocarpa, Zanthoxylum rhetsa, Sterculia striatiflora have scattered and very less population in the study area. Local extinction of a species may have far reaching implications which includes ecological imbalances in an area (Lyngdoh et al. 2016). Therefore knowledge on the availability of wild resources as well as their status is crucial at this point of time and for any locality.

\section{CONCLUSION}

This paper documents the diversity of wild fruits in East Siang district of Arunachal Pradesh. More similar studies are needed to be done in the entire North-eastern India. Some of the wild fruits could be produced through cultivation and could be commercialized. For this proper awareness on the existing fruit diversity and their importance need to be generated so that people can understand the potential of these fruits (Singh \& Asha 2017). The study shows that fruit are not only used for food and medicinal purposes but are used in many other purposes viz. sticky mesocarp of Cordia dichotoma as glue for light materials like paper; dried fruits of Sapindus mukorossi used as detergent to remove dart from ornaments and woolen clothes; Microtropis discolor, Casearia vareca, Macaranga peltata are used as bait; fruit paste of Zanthoxylum rhetsa and Gynocardia odorata are used for stupefying fishes and ripe open fruits of Sterculia striatiflora and Sterculia lanceolata var. coccinea are hung on the door to prevent the entry of evil spirits. Use of fruits has lesser impact on the plant population as in compared with use of wood, bark or rootstock.

\section{Acknowledgements}

The authors are thankful to Mr. Ojar Taku and Mr. Gyan Taram for their kind help during the field survey. Authors are also grateful to Prof. A.P. Das and Mr. Dipankar Borah for their help in the identifications of plant species and help in improving contents of the manuscript. The authors deeply acknowledge the cooperation rendered by the local (Adi) community of East Siang District during ethno-botanical study. The corresponding author (HT) is thankful to the DBT GoI for funding support to present studies through DBT Twinning Programme for NER vide grant No.: BT/PR17065/NER/95/401/2015 dated $13 / 02 / 2017$.

\section{LITERATURE CITED}

Angami, A.; Gajurel, P.R.; Rethy, P.; Singh, B. \& Kalita, S.K. 2006. Status and potential of wild edible plants of Arunachal Pradesh. Indian Journal of Traditional Knowledge 5(4):541 - 550.

Bharali, S. \& Khan, M.L. 2011. Climate change and its impact on biodiversity; some management options for mitigation in Arunachal Pradesh. Current Science 101 (7): 855-860. 
Chakravarty, S.; Suresh, C.P.; Puri, A.\&Shukla, G. 2012. North-East India, the Geographical Gateway of India's Phytodiversity. Indian Forester 138 (8):702 - 709.

Chowdhery, H.J.; Giri, G.S. \& Pramanik, A. 2009. Materials for the Flora of Arunachal Pradesh. Vol. III. Botanical Survey of India, Calcutta.

Das, A.K. 1986. Ethnobotany of East Siang District of Arunachal Pradesh. PhD thesis, University of Gauhati, Guwahati (unpublished).

Giri, G.S.; Pramanik, A. \& Chowdhery, H.J. 2008. Materials for the Flora of Arunachal Pradesh. Vol. II. Botanical Survey of India, Kolkata.

Hajra, P.K.; Verma, D.M. \&Giri, G.S. 1996. Materials for the Flora of Arunachal Pradesh. Vol. I. Botanical Survey of India, Calcutta.

Hooker, J.D. 1872-1897. Flora of British India, Vols. 1-7. L. Reeve \& Co Ltd, Ashford, Kent. London.

IUCN 2020. The IUCN Red List of Threatened Species. Version 2020-1.https:// www.iucnredlist.org [Accessed on 18 March 2020]

Jha, S.D. 1985. The wealth of Arunachal Pradesh. Mittal publication, Delhi.

Kanjilal, U.N; Das, A.; Kanjilal, P.C.; Purkaystha, C.; De, R.N. \&Bor, N.L. 1934 - 1940. Flora of Assam,Vols. I - V. Govt of Assam Press, Shillong.

Kanwal, K.S. \& Lodhi, M.S. 2018. Climate change impact on plant biodiversity of Arunachal Pradesh Biodiversity of Arunachal Himalaya: A Review. Bulletin of Arunachal Forest Research 33(2): $15-26$

Krithika, S.; Suvendu, M. \& Vasulu, T.S. 2008. A Microsatellite Guided Insight into the Genetic Status of Adi, an Isolated Hunting-Gathering Tribe of Northeast India. PLoS ONE $3(7): 1-11$

Kumar, S. \& Chaudhry, P. 2015. Ecosystem services valuation of the forest of Arunachal Pradesh State, India. Braxilian Journal of Biological Sciences 2(4): 369 - 375.

Lungphi, P.; Wangpan, T. \& Tangjang, S. 2018. Wild edible plants and their additional uses by the Tangsa community living in the Changlang district of Arunachal Pradesh, India Pleione 12(2): 151 - 164. https//:doi: 10.26679/Pleione.12.2.2018.151-164

Lyngdoh, N.; Piloo, N.; Gab, T.; Kumar, M. \& Pandey, A.K. 2016. Wild edible fruit tree resources of Arunachal Pradesh, North East India.Journal of Applied and Natural Science $8(2): 883-889$

Martin, G.J. 2008. Ethnobotany: A Methods Manual, People and Plants Conservation Series. Earthscan, UK and US A: $10-160$.

Prakash, D.; Upadhyay, G.; Gupta, C.; Pushpangadan, P. \& Singh, K. K. 2012. Antioxidant and free radical scavenging activities of some promising wild edible fruits. International Food Research Journal 19 (3): 1109 - 1116.

Singh, A.V \& Asha, H. 2017. Wild Edible Fruits of Arunachal Pradesh. International Journal of Innovative Research in Science, Engineering and Technology 6(6):12203-12209. doi:10.15680/ IJIRSET.2017.0606285

Taram, M.; Borah, D.; Rubu, R. \& Tag, H. 2018. Wild food plant resources of Komkar Adi Tribe of Upper Siang District in Arunachal Pradesh, India. Bulletin of Arunachal Pradesh Forest Research 33(2): 27 - 35.

Yanka, H.; Rubu, R.; Sanjib, K. D.; Tridip, J. D.; Dipayan, P. Debmalya, D.G.; Pallabi, K. H.; Sapana, B. Chandraiah, G.; Ananthan, R.; Sanjay, J. \& Tag, H. 2019. A brief cross- 
108 Ethnic uses of wild fruits by Adi tribe

cultural ethnobotanical note on the Abotani tribes of Arunachal Pradesh, India. Pleione 13(2): $69-283$.

Yumnam, J.Y.; Bhuyan, S.I.; Khan, M.L. \&Tripathi, O.P. 2011. Agro-diversity of East Siang-Arunachal Pradesh, Eastern Himalaya. Asian Journal of Agricultural Sciences $3(4): 317-326$.

Website accessed:

www.plantsoftheworldonline.org [Accessed on 17 April 2020]

http://www.worldfloraonline.org. [Accessed on 17 March 2020] 